\title{
ESTABILIDADE DE CULTIVARES DE MILHO-PIPOCA EM DIFERENTES AMBIENTES, NO ESTADO DE MINAS GERAIS
}

\author{
RENZO GARCIA VON PINHO ${ }^{1}$, ANDRÉ BRUGNERA², CLESO ANTONIO PATTO PACHECO ${ }^{3}$, \\ MAXIMILIAN DE SOUZA GOMES ${ }^{4}$
}

\author{
${ }^{1}$ Prof. Dr. do Depto de Agricultura, Universidade Federal de Lavras. Caixa Postal 37, CEP 37200-000 Lavras, MG. E- \\ mail: renzo@ufla.br (autor para correspondência) \\ ${ }^{2}$ Eng $^{\circ}$ Agr $^{\circ}$, Mestre em Genética e Melhoramento de Plantas, DBI, Universidade Federal de Lavras. Caixa Postal 37, \\ CEP 37200-000 Lavras, $M G$ \\ ${ }^{3}$ Pesquisador, Embrapa Milho e Sorgo. Caixa Postal 151, CEP 35701-970 Sete Lagoas, MG \\ ${ }^{4} \mathrm{Eng}^{\circ} \mathrm{Agr}{ }^{\circ}$, Doutorando em Genética e Melhoramento de Plantas, DBI, Universidade Federal de Lavras. Caixa Postal \\ 37, CEP 37200-000 Lavras, $M G$
}

Revista Brasileira de Milho e Sorgo, v.2, n.1, p.53-61, 2003

\begin{abstract}
RESUMO - O desempenho das cultivares de milho-pipoca varia com os ambientes, de modo que uma cultivar dificilmente é a melhor em todas as condições de cultivo. A resposta diferenciada das cultivares à variação ambiental denomina-se interação genótipos por ambientes. Este trabalho teve como objetivo estimar a adaptabilidade e estabilidade de cultivares de milho-pipoca quando estas foram submetidas a diferentes doses de adubação de semeadura, em diferentes locais e anos agrícolas, no estado de Minas Gerais. Foram avaliadas oito cultivares de milho-pipoca, utilizando duas doses de adubação de semeadura, em Lavras, Ijaci e Sete Lagoas, nos anos agrícolas de 2000/01 e 2001/02, perfazendo 12 ambientes. Em um dos experimentos de cada local foram utilizados $250 \mathrm{~kg} \cdot \mathrm{ha}^{-1}$ da formulação $8(\mathrm{~N}): 28\left(\mathrm{P}_{2} \mathrm{O}_{5}\right)$ : $16\left(\mathrm{~K}_{2} \mathrm{O}\right)$ na adubação de semeadura, e no outro experimento, $500 \mathrm{~kg} \cdot \mathrm{ha}^{-1}$ da mesma formulação. A adubação de cobertura foi semelhante para ambos os experimentos. O delineamento experimental para cada experimento foi o de blocos casualizados, com quatro repetições. Cada experimento foi considerado um ambiente, uma vez que foram conduzidos em diferentes locais, doses de adubação e anos agrícolas. Os resultados obtidos em todos os experimentos foram submetidos à análise de variância conjunta, considerando o peso de grão (kg.ha-1) e a capacidade de expansão $\left(\mathrm{ml} . \mathrm{g}^{-1}\right)$. Os parâmetros que avaliaram a adaptabilidade e estabilidade foram estimados a partir das metodologias de Eberhart e Russell (1966) e Annicchiarico (1992). As cultivares diferiram quanto à adaptabilidade e estabilidade para o peso de grão, não sendo observado o mesmo para a capacidade de expansão. As cultivares Zélia, IAC 112 e Ames-4198 apresentaram os melhores resultados para a produção de grãos, 2.710, 2.728 e $2.696 \mathrm{~kg} \cdot \mathrm{ha}^{-1}$ e capacidade de expansão, 28,6, 27,6 e 27,5 ml.g-1 ${ }^{-1}$, respectivamente, tanto para ambientes favoráveis como desfavoráveis. Esses cultivares apresentaram comportamento previsível e alto índice de confiança na recomendação para as regiões avaliadas.
\end{abstract}

Palavras chaves: Zea mays, cultivares, milho-pipoca, interação genótipos x ambientes.

\section{STABILITY OF POPCORN CULTIVARS TO DIFFERENT ENVIRONMENTS IN THE STATE OF MINAS GERAIS}

\begin{abstract}
The performance of popcorn cultivars varies from one environment to another. Therefore, one cultivar alone can hardly ever be considered the best for all tilling conditions. The different response of cultivars in relation to the environment is known as genotype $\mathrm{x}$
\end{abstract}


environment interaction. The purpose of this work was to estimate the adaptability and stability of popcorn cultivars as they underwent stimulated environment seed sowing fertilizing rates at different sites and harvesting years in the estate of Minas Gerais, southeastern Brazil. Eight popcorn cultivars were evaluated at two seed sowing fertilizing rates in the cities of Lavras, Ijaci and Sete Lagoas, during the harvesting years of 2000/01 and 2001/02 covering 12 different environments. Each site had two distinct experiments, the first one consisted of applying 250 kg.ha-1 of $8(\mathrm{~N}): 28\left(\mathrm{P}_{2} \mathrm{O}_{5}\right): 16\left(\mathrm{~K}_{2} \mathrm{O}\right)$ during seed sowing and the second one received 500 kg.ha ${ }^{-1}$ of the same fertilizing rate formula. Top dressing was similar for both experiments. A randomized block design with four repetitions was used in both cases. Each experiment was considered as a different environment as they were carried out at different locations, fertilizing rate and harvesting years. The experiments were submitted to an assembled variance analyses, where grain weight $\left(\mathrm{Kg}_{\mathrm{g}} \mathrm{ha}^{-1}\right)$ and expansion capacity $\left(\mathrm{ml} \mathrm{g}^{-1}\right)$ were taken into account. The parameters which evaluated the adaptability and stability were estimated by the Eberhart and Russell (1996) and Annicchiarico (1992) methods. The popcorn cultivars, differed in grain weight, but not in expansion capacity. Zélia, IAC-112 and Ames-4198 cultivars presented the best grain production and CE results, namely 2710,2728 and $2696 \mathrm{~kg}^{-h^{-1}} ; 28.6,27.6$ and $27.5 \mathrm{ml} . \mathrm{g}^{-1}$, respectively. These results were found at both favorable and unfavorable conditions, showing that these cultivars are highly indicated for the regions where they were tested.

Key words: Zea mays, cultivars, popcorn, genotype x environment interaction.

A cultura do milho-pipoca é típica do continente americano e utilizada exclusivamente para o consumo humano. Por isso, tem-se observado cada vez mais a preocupação com a qualidade. O mercado, no Brasil, encontra-se em expansão, constituindo uma boa opção econômica para os produtores.

Há vários fatores que afetam a eficiência na produção do milho-pipoca. Entre eles, a escolha da cultivar tem um grande destaque. Inúmeros relatos são encontrados na literatura sobre a diferença entre cultivares de milho-pipoca com relação à produção de grãos e capacidade de expansão (Galvão et al., 2000; Gokmen et al., 2001; Burak e Broccoli, 1999).

O desempenho das cultivares varia, normalmente, com os ambientes, de modo que uma cultivar dificilmente é a melhor em todas as condições de cultivo. A resposta diferenciada das cultivares à variação ambiental denomina-se interação genótipos por ambientes. Isso significa que os efeitos genéticos e ambientais não são independentes.
Há alternativas para atenuar os efeitos da interação genótipos por ambientes. Uma das mais empregadas é a identificação de cultivares que apresentem maior produção de grãos e boa capacidade de expansão, por meio de diversas metodologias de adaptabilidade e estabilidade.

No Brasil, o estudo da adaptabilidade e estabilidade de cultivares de milho-pipoca não recebeu a mesma atenção dada ao milho comum. Isso se deve principalmente ao número limitado de cultivares comerciais, instituições e melhoristas envolvidos com esse tipo de milho especial. Existem relatos de que os fatores ambientais afetam a produção de grãos e a capacidade de expansão (Vendruscolo et al., 2001; Nunes, 2002). Nesses trabalhos, não só tem sido quantificado o efeito da interação para a expressão fenotípica como também procurado alternativas para atenuar seu efeito.

Este trabalho foi conduzido com o objetivo de estimar a adaptabilidade e a estabilidade de cultivares de milho-pipoca, submetidas a diferentes condições ambientais, no Estado de Minas Gerais. 


\section{Material e Métodos}

Foram utilizadas oito cultivares de milho-pipoca provenientes de diferentes empresas e/ou instituições e originárias de programas de melhoramento nacionais ou internacionais (Tabela 1). Os experimentos foram conduzidos nos seguintes locais de Minas Gerais: Lavras, Ijaci e Sete Lagoas.

Nos três locais, na segunda quinzena de novembro, nos anos agrícolas 2000/01 e 2001/2002, foram instalados dois experimentos, em regime de sequeiro, totalizando-se 12 experimentos. Em um dos experimentos de cada local foram utilizados, na adubação de semeadura, $250 \mathrm{~kg} \cdot \mathrm{ha}^{-1}$ da formulação $8(\mathrm{~N}): 28\left(\mathrm{P}_{2} \mathrm{O}_{5}\right): 16\left(\mathrm{~K}_{2} \mathrm{O}\right) \mathrm{e}$, no outro experimento, 500 kg.ha ${ }^{-1}$ da mesma formulação. A adubação de cobertura foi semelhante para ambos os experimentos. O delineamento experimental para cada experimento foi o de blocos casualizados com quatro repetições. As parcelas experimentais constituíram-se de quatro fileiras de cinco metros, espaçadas de $0,80 \mathrm{~m}$, com área total de $16 \mathrm{~m}^{2}$ e área útil de $8 \mathrm{~m}^{2}$ (duas linhas centrais).

Foram avaliados o peso de grãos e a capacidade de expansão (CE). Os dados referentes ao peso de grãos foram corrigidos para umidade-padrão de $13 \%$ e transformados para kg.ha ${ }^{-1}$. A CE foi obtida a partir da razão entre o volume de pipoca expandida e o peso de grãos. Para isso, foi coletada uma amostra de cinco espigas de cada parcela, que foram debulhadas manualmente para diminuir os danos físicos. A capacidade de expansão foi determinada em laboratório, pela relação volume de pipoca/peso de grãos $\left(\mathrm{ml} . \mathrm{g}^{-1}\right)$, utilizando-se uma amostra de peso equivalente a $30 \mathrm{ml}$ de grãos. Para o pipocamento, foi utilizada uma pipoqueira de ar quente, a Hot Air Popcorn Pumper H7340, da Proctor Sílex, com 1250 watts de potência. Os grãos foram colocados no recipiente da pipoqueira, sem óleo, quando a temperatura atingiu $100^{\circ} \mathrm{C}$, e mantidos por trinta segundos. O volume da pipoca expandida foi medido em proveta graduada de 1.000 ml (Sawazaki, 1996).

Cada experimento foi considerado um ambiente, uma vez que foram conduzidos em diferentes locais, doses de adubação e anos agrícolas. Os dados assim obtidos para peso de grãos e capacidade de expansão foram submetidos à análise de variância por ambiente e à análise de variância conjunta, envolvendo todos os ambientes, com a finalidade de detectar a interação cultivares e ambientes (Ramalho et al., 2000). A partir dos resultados médios, foram estimados os parâmetros que avaliam a adaptabilidade e a estabilidade, utilizando-se o procedimento de Eberhart e Russel (1966), pelo seguinte modelo:

TABELA 1. Características das oito cultivares de milho-pipoca utilizadas nos experimentos. UFLA, Lavras, MG, 2002.

\begin{tabular}{llll}
\hline Cultivares & Tipo & Instituição & Origem \\
\hline BRS - Ângela & Variedade & EMBRAPA & Brasil \\
DFT -2 & Variedade & UFV & Brasil \\
RS -20 & Variedade & AGROESTE & Brasil \\
ZÉLIA & Híbrido Triplo & PIONEER & Brasil \\
UFLA -1 & Variedade & UFLA & Brasil \\
AMES-4198 & Híbrido simples & EMP. BRAS. de POPCORN & EUA \\
UFLA -2 & Variedade & UFLA & Brasil \\
IAC -112 & Híbrido simples mod. & IAC & Brasil \\
\hline
\end{tabular}


$Y_{i j}=\beta_{0 i}+\beta_{1 i} I_{j}+\delta_{i j}+\varepsilon_{i j,}$

Emque:

$\mathrm{Y}_{\mathrm{ij}}$ : valor observado do genótipo i, no ambiente j e na repetição $\mathrm{k} ; \beta_{0 \mathrm{i}}$ : média geral do genótipo $i ; \beta_{1 \mathrm{i}}$ : resposta linear do genótipo i a variação ambiental; $\mathrm{I}_{\mathrm{j}}$ : índice ambiental (diferença entre a média de cada ambiente e a média geral); $\delta_{\mathrm{ij}}$ : desvio da regressão do genótipo i no ambiente $\mathrm{j} ; \varepsilon_{\mathrm{ij}}$ : erro experimental médio.

Foi estimado também o risco de adoção de uma determinada cultivar, utilizando o procedimento de Annicchiarico (1992). Para isso, foi estimada a percentagem $\left(\mathrm{Y}_{\mathrm{i}}\right)$ de cada cultivar em relação a média dos ambientes. Posteriormente, foi estimado o desvio-padrão fenotípico percentual $\left(\mathrm{s}_{\mathrm{i}}\right)$ de cada cultivar. A partir desses dados, foi estimado o índice de confiança $\left(\mathrm{W}_{\mathrm{i}}\right.$ ) pela expressão: $\mathrm{W}_{\mathrm{i}}=\mathrm{Y}_{\mathrm{i} .}-\mathrm{Z}_{(1-\alpha)}$ $\mathrm{s}_{\mathrm{i}}$ em que: $\mathrm{W}_{\mathrm{i}}$ : índice de confiança $(\%) ; \mathrm{Y}_{\mathrm{i}}$ : média geral do genótipo i em porcentagem; Z: percentil $(1-\alpha)$ da função de distribuição normal acumulada; a: nível de significância; $\mathrm{s}_{\mathrm{i}}$ : desvio padrão dos valores percentuais.

\section{Resultados e Discussão}

A acurácia experimental, avaliada pelo coeficiente de variação (CV), variou entre as características estudadas. Observou-se maior $\mathrm{CV}$ para o peso de grãos (20\%) do que para a capacidade de expansão (19,1\%). Pela análise de variância conjunta, foram constatados efeitos significativos para cultivares, ambientes e interação cultivares e ambientes para peso de grãos (Tabela 2). No caso da capacidade de expansão (CE), apenas as fontes de variação cultivares e ambientes foram significativas.

Constata-se, pela Tabela 3, que o peso de grãos médio variou de $1.562 \mathrm{~kg} \cdot \mathrm{ha}^{-1}$, em Sete Lagoas/safra 2000/2001, com a menor dose de adubação, a 3.327 kg.ha-1 ${ }^{-1}$ em Ijaci/safra 2000/2001, com a maior dose de adubação. A amplitude da variação para a capacidade de expansão foi menor.
Ela variou de 22,3 ml.g-1 , em Sete Lagoas/safra 2000/ 2001, com a dose maior de adubação, a 31,6 ml.g-1 em Lavras/safra, 2001/2002, com a dose maior de adubação.

TABELA 2. Resumo da análise de variância conjunta do peso de grãos (PROD), em kg.ha-1 ${ }^{-1}$ e capacidade de expansão (CE), em $\mathrm{ml} \mathrm{g}^{-1}$, referente às cultivares de milho-pipoca analisadas em doze ambientes, nos anos agrícolas de 2000/2001 e 2001/2002. UFLA, Lavras, MG, 2002.

\begin{tabular}{lccc}
\hline \multirow{2}{*}{ FV } & GL & \multicolumn{2}{c}{ Quadrados médios } \\
\cline { 3 - 4 } & 36 & PROD & CE \\
\hline B/A & 7 & $917682,41^{* *}$ & $145,96^{* *}$ \\
Cultivares (C) & 11 & $8688990,96^{* *}$ & $290,99^{* *}$ \\
Ambientes (A) & 77 & $660211,67^{* *}$ & $45,22 \mathrm{~ns}$ \\
C x A & 252 & 288398,84 & 23,66 \\
Resíduo & \multicolumn{3}{c}{2600,00} \\
\hline Média & 20,65 & 26,20 \\
\hline CV (\%)
\end{tabular}

** Significativos a $1 \%$ de probabilidade, pelo teste de F.

Observando-se as médias dos ensaios e comparando-se os níveis de adubação dentro de cada local, dificilmente será detectada significância do nível de adubação para a capacidade de expansão. Por outro lado, a adubação influenciou no peso de grãos das cultivares, tendo a produção sido, em média, $10 \%$ superior nos experimentos de maior dose de adubação. Essa superioridade não foi observada em Sete Lagoas no ano agrícola de 2000/ 2001.

A variação na produção de grãos entre os ambientes evidencia a instabilidade nas condições climáticas durante o período estudado. Dos doze ambientes, sete foram classificados como favoráveis e cinco como não favoráveis. É importante enfatizar que a separação dos ambientes favoráveis dos desfavoráveis foi independente das duas doses de adubação. Os ambientes não favoráveis foram os 
TABELA 3. Índices ambientais, médias de peso de grãos (PROD), em kg.ha- ${ }^{-1}$, e capacidade de expansão (CE), em ml.g-1 considerando a média das oito cultivares de milho-pipoca avaliadas nos doze ambientes. UFLA, Lavras, MG, 2002.

\begin{tabular}{|c|c|c|c|c|}
\hline \multirow[b]{2}{*}{ Ambientes } & \multicolumn{2}{|c|}{ PROD. } & \multicolumn{2}{|c|}{$\mathrm{CE}$} \\
\hline & $\begin{array}{c}\text { Índice } \\
\text { ambiental }\end{array}$ & Médias & $\begin{array}{c}\text { Índice } \\
\text { ambiental }\end{array}$ & Médias \\
\hline \multicolumn{5}{|l|}{ LAVRAS: } \\
\hline $2000 / 2001 /$ Dose menor ${ }^{1}$ & 330,59 & 2911 & $-1,76$ & 24,5 \\
\hline $2000 / 2001 /$ Dose maior ${ }^{2}$ & 675,96 & 3256 & $-1,06$ & 25,2 \\
\hline 2001/2002 / Dose menor & $-316,28$ & 2264 & 4,38 & 30,6 \\
\hline 2001/2002 / Dose maior & 67,90 & 2647 & 5,35 & 31,6 \\
\hline \multicolumn{5}{|l|}{ IJACI: } \\
\hline 2000/2001 / Dose menor & 141,59 & 2722 & $-2,98$ & 23,3 \\
\hline 2000/2001 / Dose maior & 747,46 & 3327 & $-2,26$ & 24,0 \\
\hline 2001/2002 / Dose menor & 57,71 & 2638 & 2,80 & 29,0 \\
\hline 2001/2002 / Dose maior & 224,59 & 2805 & 2,45 & 28,7 \\
\hline \multicolumn{5}{|l|}{ SETE LAGOAS: } \\
\hline 2000/2001 / Dose menor & $-64,78$ & 2515 & $-0,69$ & 25,6 \\
\hline 2000/2001 / Dose maior & $-236,65$ & 2343 & $-4,00$ & 22,3 \\
\hline 2001/2002 / Dose menor & $-1018,28$ & 1562 & $-1,93$ & 24,3 \\
\hline 2001/2002 / Dose maior & $-609,03$ & 1971 & $-0,28$ & 26,0 \\
\hline Médias & - & 2600 & - & 26,3 \\
\hline
\end{tabular}

${ }^{1}$ Dose menor: $250 \mathrm{~kg}$. ha-1 da formulação $8(\mathrm{~N}): 28\left(\mathrm{P}_{2} \mathrm{O}_{5}\right): 16\left(\mathrm{~K}_{2} \mathrm{O}\right)$ de semeadura.

${ }^{2}$ Dose maior: $500 \mathrm{~kg}$. ha ${ }^{-1}$ da formulação $8(\mathrm{~N}): 28\left(\mathrm{P}_{2} \mathrm{O}_{5}\right)$ : $16\left(\mathrm{~K}_{2} \mathrm{O}\right)$ de semeadura.

experimentos realizados em Lavras, com a menor dose de adubação, no ano agrícola de 2001/2002, e todos os experimentos conduzidos em Sete Lagoas (Tabela 3). Possivelmente, as condições climáticas menos favoráveis para o cultivo do milho-pipoca e a menor altitude de Sete Lagoas, quando comparadas às dos outros locais, foi a principal razão para que todos os experimentos conduzidos em Sete Lagoas tenham sido classificados como ambientes não favoráveis.
Para a capacidade de expansão, a variação verificada entre ambientes permitiu dividí-los em quatro ambientes favoráveis e oito desfavoráveis (Tabela 3). Os ambientes favoráveis foram os de Lavras, no ano agrícola 2001/2002, para as duas doses de adubação de semeadura, e Ijaci, no ano agrícola de 2001/2002, também para as duas doses. É importante enfatizar que a separação dos ambientes favoráveis dos desfavoráveis foi independente das duas doses de adubação. As maiores 
diferenças entre ambientes deveram-se aos locais e anos. Mais uma vez, todos os experimentos de Sete Lagoas foram considerados como ambientes não favoráveis.

As cultivares IAC-112, Zélia, Ames-4198, UFLA-1 e BRS-Angela produziram acima da média geral (2.600 kg.ha-1 $)$, mostrando o bom desempenho nas regiões de Minas Gerais (Tabela 4). Esses resultados estão acima da média de 2.100 $\mathrm{kg}$.ha- ${ }^{-1}$ da cultivar com melhor desempenho, na região Centro-sul do Brasil, avaliado por Vendruscolo et al. (2001). Outros autores obtiveram bons rendimentos de milho-pipoca com diferentes germoplasmas. Galvão et al. (2000) obtiveram, para o melhor híbrido experimental de milho-pipoca, a média de $5.828 \mathrm{~kg}$.ha- ${ }^{-1}$. Coimbra (2000), em condições irrigadas, obteve o rendimento médio de 4.924 kg.ha- ${ }^{-1}$ das progênies da população DFT1 de milho-pipoca e as médias das testemunhas foram de $5.400 \mathrm{~kg} \mathrm{ha}^{-1}$ (Zélia), $5.311 \mathrm{~kg} \cdot \mathrm{ha}^{-1}($ IAC-112) e $5.100 \mathrm{~kg} \cdot \mathrm{ha}^{-1}$ (RS-20).
As cultivares BRS-Angela, RS-20 e UFLA1 apresentaram estimativas do coeficiente de regressão que não diferiram estatisticamente da unidade $(\mathrm{P} \leq 0,01)$, evidenciando adaptação a ambientes favoráveis e desfavoráveis, ou seja, de ampla adaptabilidade. As cultivares DFT-2 e UFLA-2 apresentaram valor de $\hat{\beta} \mathbf{i}$ significativamente inferior a um, sendo adaptadas a ambientes desfavoráveis. Por outro lado, as cultivares Zélia, Ames-4198 e IAC112 apresentaram $\hat{\beta} \mathrm{i}$ significativamente superior a um, sendo considerado adaptadas a ambientes favoráveis. A estimativa do $\hat{\beta} \mathrm{i}$ do cultivar DFT-2 e da UFLA-2 não foi considerada de credibilidade, pois os coeficientes de determinação das equações de regressão foram extremamente baixos, não sendo confiáveis. Dessa forma, o modelo linear proposto por Eberhart e Russell (1966) não explicou o comportamento dessas cultivares.

Com relação às estimativas das variâncias dos desvios da regressão ( $\left.\hat{\sigma}_{d i}^{2}\right)$, as cultivares Zélia, Ames-4198, UFLA-2 e IAC-112 apresentaram $\hat{\sigma}_{d i}^{2}$ não-significativos, demonstrando estabilidade de

TABELA 4. Média de peso de grãos (PROD), em kg.ha-1, coeficientes de regressão ( $\hat{\beta} \mathrm{i}$ ), variâncias dos desvios da regressão( $\left.\hat{\sigma}_{d i}^{2}\right)$, coeficiente de determinação $\left(\hat{\mathrm{R}}^{2}(\%)\right.$ e índice de confiança $\left(\mathrm{W}_{\mathrm{i}}\right)$ das cultivares de milho-pipoca. UFLA, Lavras, MG, 2002.

\begin{tabular}{lccccc}
\hline Cultivares & Médias & $\hat{\beta} i$ & $\hat{\sigma}^{2} d i$ & $\hat{\mathrm{R}}^{2}(\%)$ & $\mathrm{W}_{\mathrm{i}}$ \\
\hline BRS-Angela & $2603 \mathrm{a}$ & $1,01 \mathrm{~ns}$ & $677811+$ & 63 & 101,15 \\
DFT-2 & $2430 \mathrm{~b}$ & $0,24 * *$ & $972202++$ & 6 & 97,90 \\
RS-20 & $2412 \mathrm{~b}$ & $0,70 \mathrm{~ns}$ & $279688++$ & 67 & 92,27 \\
Zélia & $2710 \mathrm{a}$ & $1,53 * *$ & $335922 \mathrm{~ns}$ & 88 & 102,87 \\
UFLA-1 & $2628 \mathrm{a}$ & $0,99 \mathrm{~ns}$ & $783208++$ & 58 & 101,62 \\
Ames 4198 & $2696 \mathrm{a}$ & $1,38 *$ & $430766 \mathrm{~ns}$ & 83 & 102,94 \\
UFLA-2 & $2486 \mathrm{~b}$ & $0,64 *$ & $411109 \mathrm{~ns}$ & 53 & 97,23 \\
IAC-112 & $2728 \mathrm{a}$ & $1,47 * *$ & $69235 \mathrm{~ns}$ & 97 & 103,99 \\
\hline
\end{tabular}

Médias seguidas pelas mesmas letras pertencem ao mesmo agrupamento, de acordo com o teste de Scott-Knott, a 5\% de probabilidade.

*, **- diferem significativamente de 1 pelo teste $\mathrm{t}$, a $5 \%$ e $1 \%$ de probabilidade.

${ }^{+},++$-significativos pelo teste de $\mathrm{F}$, a $5 \%$ e $1 \%$ de probabilidade.

ns - não-significativo. 
comportamento ou previsibilidade em relação às variações do ambiente. As cultivares Zélia, Ames 4198 e IAC 112 destacaram-se entre as mais produtivas e adequadas para ambientes favoráveis, apresentando alta estabilidade.

De acordo com a metodologia de Eberhart e Russell (1966), é possível identificar três tipos de cultivares. Dois destes são adequados a ambientes específicos, favoráveis ou desfavoráveis. Normalmente, as cultivares para ambientes favoráveis $(\beta>1)$ são adequadas para o aumento da aplicação de insumos. Para ambientes desfavoráveis $(\beta<1)$, as cultivares são adequadas à condição de utilização de poucos insumos ou baixa tecnologia. $O$ terceiro tipo de cultivar é o não específico, sendo ideal para o uso de baixo e alto insumos, pois apresenta ampla adaptabilidade $(\beta=1)$, sendo capaz de responder moderadamente ao estímulo do ambiente. Para os três tipos de cultivares, a estabilidade deve ser a mais alta, para que o comportamento seja previsível, mesmo com alteração do ambiente em que a cultivar será futuramente utilizada.

As cultivares BRS-Angela e UFLA-1 apresentaram média de rendimento de grãos superior à média geral, adaptabilidade ampla, demonstrando capacidade satisfatória no aproveitamento dos estímulos dos ambientes, sendo, portanto, adequadas para ambientes não específicos. A cultivar RS-20 também apresentou adaptabilidade ampla, no entanto, sua produtividade foi menor que a média de rendimento de grãos dos experimentos. As cultivares DFT-2 e RS-20 apresentaram características indesejáveis, como a média de peso de grãos inferior à média geral e instabilidade, apesar da adaptabilidade ampla para o RS-20. A cultivar UFLA-2 apresentou média de rendimento de grãos pouco inferior à média geral e alta estabilidade; porém, demonstrou adaptação a ambientes desfavoráveis. As cultivares Zélia, Ames-4198 e IAC-112 destacaram-se entre as mais produtivas e adequadas para ambientes favoráveis, apresentando alta estabilidade.
Utilizando o método que avalia o índice de confiança $\left(\mathrm{W}_{\mathrm{i}}\right)$ (Annicchiarico, 1992), tem-se a vantagem de recomendar as cultivares considerando o risco de apresentarem desempenho abaixo de um dado padrão, como, por exemplo, a média geral. Quanto maior o índice de confiança de uma dada cultivar, tanto menor será a sua probabilidade de insucesso. Avaliando-se as cultivares por esse método, destacaram-se, para peso de grãos, as cultivares IAC-112, Ames 4198, Zélia, UFLA-1 e BRSAngela. Essas apresentaram-se com $75 \%$ de probabilidade de, na pior das hipóteses, ficarem 3,99\%, $2,94 \%, 2,87 \%, 1,62 \%$ e $1,15 \%$, respectivamente, acima da média dos ambientes (Tabela 4).

Como comentado anteriormente, para o caráter capacidade de expansão, não foi detectada diferença significativa na interação genótipos e ambientes. Apesar da não significância para essa fonte de variação, optou-se por avaliar a adaptabilidade e estabilidade das cultivares para esse caráter, para detectar os materiais mais adaptados e estáveis pelos métodos de adaptabilidade e estabilidade avaliados.

Para a capacidade de expansão, conforme preconizado pela metodologia de Eberhart \& Russel (1966), as cultivares que apresentam coeficiente de regressão igual a 1,0 são aquelas com adaptabilidade ampla ou geral. Como todos os $\hat{a}_{1 \mathrm{i}}$ não diferiram significativamente da unidade, todas as cultivares podem ser consideradas como de adaptabilidade ampla ou geral para o caráter em questão (Tabela 5). As estimativas dos $\hat{\beta} \mathrm{i}$ da maioria das cultivares não merecem credibilidade, pois os coeficientes de determinação das equações de regressão foram extremamente baixos; portanto, não confiáveis. No entanto, as cultivares IAC 112, Zélia, Ames-4198, DFT-2 e RS-20 apresentaram a média de CE superior à média geral, mostrando que as mesmas possuem, em relação ao grupo de cultivares avaliadas, estabilidade biológica, ou seja, boa CE em qualquer 
TABELA 5. Média de capacidade de expansão (CE), em $\mathrm{ml} \mathrm{g}^{-1}$, coeficientes de regressão ( $\left.\hat{\beta} \mathrm{i}\right)$, variâncias dos desvios da regressão $\left(\hat{\sigma}^{2}{ }_{d i}\right)$, coeficiente de determinação $\left(\hat{R}^{2}(\%)\right)$ e índice de confiança $\left(\mathrm{W}_{\mathrm{i}}\right)$ das cultivares de milho-pipoca. UFLA, Lavras, MG, 2002.

\begin{tabular}{lccccc}
\hline Cultivares & Médias & $\hat{\beta} i$ & $\hat{\sigma}^{2}{ }_{\mathrm{di}}$ & $\hat{\mathrm{R}}^{2}(\%)$ & $\mathrm{W}_{\mathrm{i}}$ \\
\hline BRS-Angela & $24,9 \mathrm{~b}$ & $0,85 \mathrm{~ns}$ & $26,1 \mathrm{~ns}$ & 53 & 94,89 \\
DFT-2 & $27,3 \mathrm{a}$ & $0,61 \mathrm{~ns}$ & $48,7 \mathrm{~ns}$ & 24 & 104,41 \\
RS-20 & $26,2 \mathrm{a}$ & $1,03 \mathrm{~ns}$ & $18,8 \mathrm{~ns}$ & 70 & 99,80 \\
Zélia & $28,6 \mathrm{a}$ & $1,45 \mathrm{~ns}$ & $12,3 \mathrm{~ns}$ & 87 & 106,95 \\
UFLA-1 & $24,4 \mathrm{~b}$ & $0,98 \mathrm{~ns}$ & $80,6 \mathrm{~ns}$ & 32 & 94,26 \\
Ames 4198 & $27,6 \mathrm{a}$ & $1,54 \mathrm{~ns}$ & $24,4 \mathrm{~ns}$ & 80 & 104,29 \\
UFLA-2 & $23,7 \mathrm{~b}$ & $0,39 \mathrm{~ns}$ & $37,1 \mathrm{~ns}$ & 14 & 90,77 \\
IAC-112 & $27,5 \mathrm{a}$ & $1,12 \mathrm{~ns}$ & $72,0 \mathrm{~ns}$ & 41 & 104,59 \\
\hline
\end{tabular}

Médias seguidas pelas mesmas letras pertencem ao mesmo agrupamento, de acordo com o teste de Scott-Knott, a 5\% de probabilidade.

*,**-diferem significativamente de 1 pelo teste $\mathrm{t}$, a $5 \%$ e $1 \%$ de probabilidade.

${ }^{+},++$-significativos pelo teste de F, a $5 \%$ e $1 \%$ de probabilidade.

Ns - não-significativo.

condição de ambiente. Com relação às estimativas das variâncias dos desvios da regressão $\left(\hat{\sigma}_{d i}^{2}\right)$, todas as cultivares apresentaram $\hat{\sigma}_{d i}^{2}$ não-significativo, demonstrando estabilidade de comportamento ou previsibilidade às variações do ambiente.

Esses resultados (Tabela 5) estão acima da média da cultivar com melhor desempenho na região Centro-Sul do Brasil, que foi de 21, em $\mathrm{ml} / \mathrm{ml}$ (Vendruscolo et al., 2001). Galvão et al. (2000) obtiveram valores de até $39 \mathrm{~g} \mathrm{ml}^{-1}$ para $\mathrm{CE}$, quando avaliaram híbridos experimentais e as testemunhas Zélia e IAC 112, cujos valores de CE foram, respectivamente, de 34,5 e $37,6 \mathrm{ml} \mathrm{g}^{-1}$.

Utilizando o índice de confiança $\left(\mathrm{W}_{\mathrm{i}}\right)$ obtido pela metodologia de Annicchiarico (1992), ou seja, as cultivares que se destacaram para capacidade de expansão por esse método foram Zélia, IAC-112, DFT-2, Ames-4198, que apresentaramse com $75 \%$ de probabilidade de, na pior das hipóteses, ficarem 4,95\%, 4,59\%, 4,41\% e 4,29\%, respectivamente, acima da média dos ambientes (Tabela 5).

Na cultura do milho-pipoca, a capacidade de expansão é característica extremamente importante, uma vez que, quanto maior for a capacidade de expansão da cultivar, maior será a qualidade da pipoca. No entanto, o ideal é que a cultivar apresente também bons rendimentos de grãos. Assim, considerando as médias das duas características, somente as cultivares Zélia, IAC 112 e Ames-4198 apresentaram ótimos resultados para ambas.

\section{Conclusões}

As cultivares avaliadas diferem quanto à adaptabilidade e estabilidade de produção de grãos.

As metodologias de Eberhart e Russell (1966) e Annicchiarico (1992) discriminaram as cultivares Zélia, IAC-112 e Ames-4198 como superiores quanto à produção de grãos e à capacidade de expansão, apresentando os menores riscos de baixa produtividade e capacidade de expansão.

\section{Literatura Citada}

ANNICCHIARICO, P. Cultivar adaptations and recommendation from alfafa trials in Northern Italy. Journal of Genetics \& Breeding, Rome, v.46, n.1, p.269-278, 1992. 
BURAK, R.; BROCCOLI, A. M. Genotype by environment interaction on popping expansion and yield in popcorn hybrids cultivated in Argentina. Maize Genetics Newsletter, v.74,1999. Disponível <www.agron.missouri. edu/mnl/74/ 80burak.html> Acesso: em 13 maio 2002.

COIMBRA, R. R. Seleção entre famílias de meios-irmãos da população DFT 1-Ribeirão de Milho-pipoca. 2000, 54f. Dissertação (Mestrado em Genética e Melhoramento de Plantas) - Universidade Federal de Viçosa, Viçosa.

EBERHART, S. A.; RUSSELL, W. A. Stability parameters for comparing varieties. Crop Science, Madison, v.6, n.1, p.36-40, 1966.

GALVÃO, J. C. C.; SAWAZAKI, E.; MIRANDA, G. V. Comportamento de híbridos de milho-pipoca em Coimbra, Minas Gerais, Revista Ceres, Viçosa, v.47, n.247, p.201-218, 2000.

GOKMEN, S; SENCAR, O.; SAKIN, M. A. Response of popcorn to nitrogen rates and plant densities. Journal of Agriculture and Forestry. Turkish, v.25, n.1, p.15-24, 2001.
NUNES, H. V. Comportamento, adaptabilidade e estabilidade de cultivares de milho-pipoca em diferentes épocas de semeadura. 2002. 66f. Dissertação (Mestrado em Genética e Melhoramento de Plantas) - Universidade Federal de Viçosa, Viçosa.

RAMALHO, M. A. P.; FERREIRA, D. F.; OLIVEIRA, A. C. de A experimentação em genética e melhoramento de plantas. Lavras: UFLA, 2000. 326p.

SAWAZAKI, E. Parâmetros genéticos em milho-pipoca (Zea mays L. ). 1996. 157f. Tese (Doutorado em Genética e Melhoramento de Plantas) USP/Escola Superior de Agricultura "Luiz de Queiroz", Piracicaba.

VENDRUSCOLO, E. C. G.; SCAPIN, C. A.; PACHECO, C. A. P.; OLIVEIRA, V. R. de; BRACCINI, A. L.; VIDIGAL, M. C. G. Adaptabilidade e estabilidade de cultivares de milho-pipoca na região centro-sul do Brasil. Pesquisa Agropecuária Brasileira, Brasília, v.36, n.1, p.123-30, 2001. 